\title{
GAIA Level 4 Second Trimester Spontaneous Abortion
}

National Cancer Institute

\section{Source}

National Cancer Institute. GAIA Level 4 Second Trimester Spontaneous Abortion. NCI

Thesaurus. Code C128783.

GAIA Level 4 Second T rimester Spontaneous Abortion is defined by two criteria: first, it does not qualify as either a Level 1 or a Level 2 Second T rimester Spontaneous Abortion; second, a maternal self-report or documentation in the medical record of a pregnancy loss without sufficient ultrasound or laboratory evidence to confirm. 